\title{
Chronic, nonspecific, postinfectious, retroperitoneal fibrosis and ureteral obstruction
}

\author{
Angelo Territo ${ }^{1}$, Salvatore Micali ${ }^{1}$, Antonio Manenti ${ }^{2}$, Eugenio Martorana ${ }^{1}$, Giacomo Maria Pirola ${ }^{1}$, Giampaolo Bianchi ${ }^{1}$ \\ ${ }^{1}$ Department of Urology, University of Modena and Reggio Emilia, Modena - Italy \\ ${ }^{2}$ Department of General Surgery, University of Modena and Reggio Emilia, Modena - Italy
}

\begin{abstract}
Introduction: Two cases of severe ureteral obstruction following nonspecific, postinfectious, chronic retroperitoneal fibrosis are described, which both originated by a primitive intestinal pathology.

Patients: This complication was observed in two women: first, 65 years old, submitted for ulcerative colitis to a total proctocolectomy, with ileo-pouch-anal anastomosis, complicated by an anastomotic fistula; and second, 66 years old, operated with an extended left hemicolectomy, for an adenocarcinoma of the recto-sigmoid colon complicated with a vaginal fistula. In these cases, computerized tomography demonstrated a unilateral hydronephrosis, secondary to a complete obstruction of the ureter; a subsequent nephro-ureterectomy became necessary. Histology demonstrated nonspecific inflammatory lesions.

Discussions: Postinfectious, chronic inflammation of the retroperitoneum acts on the ureteral and peri-ureteral tissues, inducing an inflammatory and then a fibrotic process.

Conclusions: We underline the opportunity of a precocious and radical treatment of every retroperitoneal infection.
\end{abstract}

Keywords: Intestinal fistula, Retroperitoneal fibrosis, Retroperitoneal infection, Ureteral obstruction

\section{Introduction}

The idiopathic retroperitoneal fibrosis, in its evolution, often arrives to encase and obstruct ureters (1). On the contrary, chronic, nonspecific, postinfectious inflammatory conditions of the retroperitoneum, originated by a primitive intestinal pathology and following an intestinal surgical procedure, usually do not cause this urological complication (2-9). The following two cases are an example of this uncommon disease.

\section{Patients}

A woman 65 years old underwent a proctocolectomy for ulcerative colitis, with an ileal pouch anal anastomosis, and a diverting ileostomy, performed for 4 months. After 2 years, the patient presented signs of recurrent pouchitis, which were treated conservatively, obtaining long periods of remission. Ten years later, at the age of 75 years, a computerized

Accepted: July 6, 2015

Published online: August 27, 2015

Corresponding author:

Angelo Territo, MD

Department of Urology

University of Modena and Reggio Emilia

Via Largo del Pozzo 71

41122 Modena, Italy

territoangelo@tiscali.it tomography (CT) demonstrated a long sinus originating from the ileal poch anal anastomosis and directed toward the presacral space, but without concomitant lesions to the kidneys, ureters, and bladder (Fig. 1A, B). The associated clinical signs of severe infection resolved after a long medical treatment. After 6 months, the patient complained of a mild pain in the right flank. An abdominal ultrasound and a subsequent CT demonstrated a right hydronephrosis, a diffuse thickening of the retroperitoneal tissue of the lower retroperitoneum and of the pelvic pre-sacral space involving and completely obstructing the right ureter in its lower third, which also appeared to be medially deviated. On the left side, at the same level, a slight narrowing of the ureter was discovered, with only a mild upper urinary tract dilatation and a preserved excretory renal function (Fig. 1C, D). No signs of residual fistulous tract at pouchgraphy were observed. An urgent right percutaneous nephrostomy was performed, followed by a subsequent nephro-ureterectomy. Histology demonstrated only signs of nonspecific chronic ureteral and peri-ureteral inflammatory lesions.

A female 63 years old, after 1 year of colonic troubles, presented an enterovaginal fistula. Colonscopy confirmed a significant stenosis at the distal sigmoid, with a biopsy positive for adenocarcinoma. CT and magnetic resonance imaging (MRI) showed a big neoplastic mass arising from the rectosigmoid junction, strictly adherent to the uterus, complicated by a fistula running toward the vagina, and by an inflammatory reaction in the right hemi-pelvis. The kidneys, the ureters, and the bladder appeared normal; no secondary hepatic or 


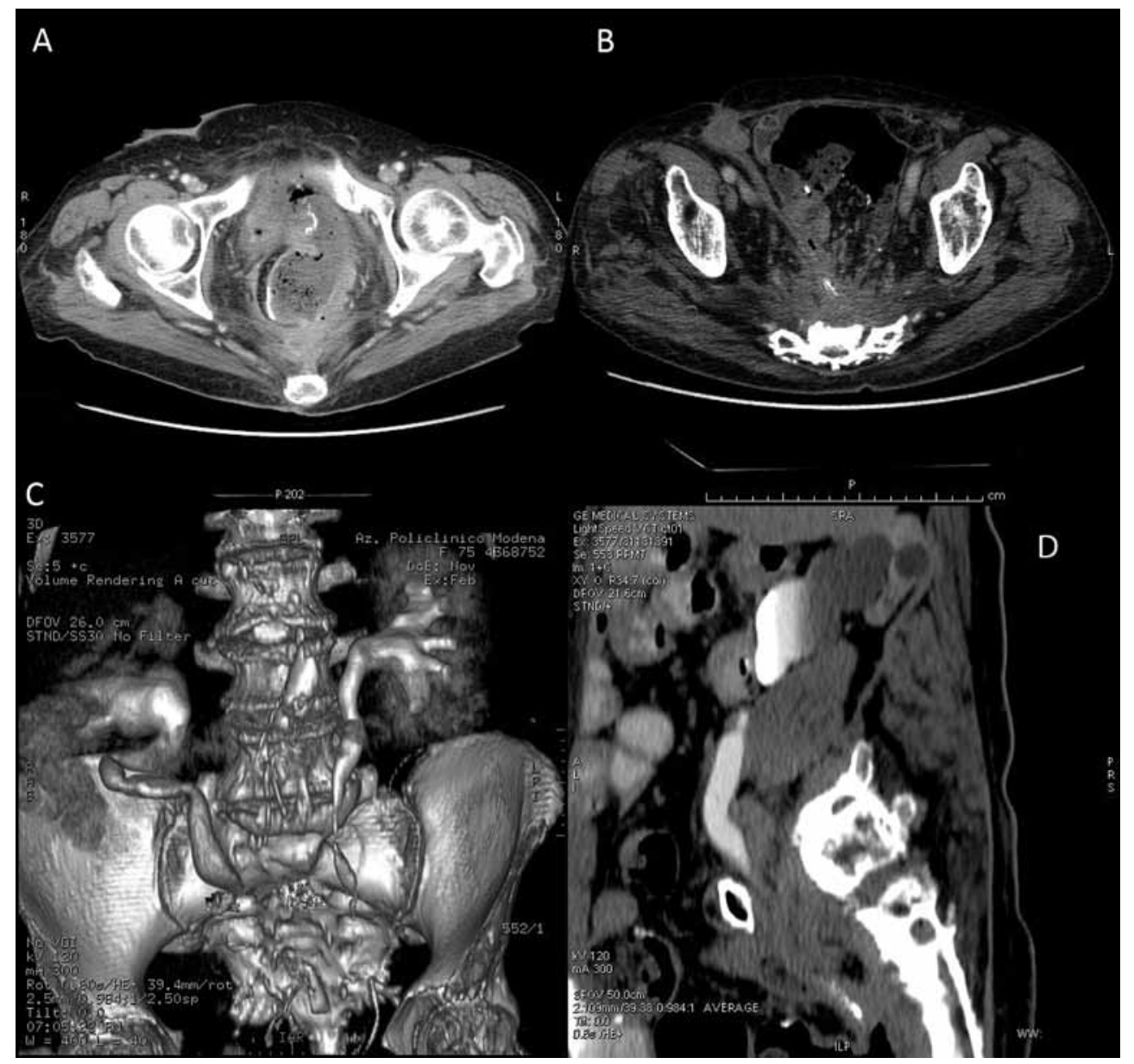

Fig. 1 - (A) Contrast-enhanced CT. The ileal reservoir occupies the small pelvis, surrounded by a hypodense inflammatory tissue. (B) Contrast-enhanced CT. Inflammatory hypodense tissue in the pre-sacral space, extended toward the right side of the retroperitoneum. (C) Contrast-enhanced CT volume rendering reconstruction. Right uretero-hydronephrosis, with medial deviation of the middle ureter. Moderate dilatation of the left renal pelvis and upper ureter, narrowed in its middle part. (D) Contrastenhanced CT sagittal reconstruction. The renal pelvis and the upper ureter are markedly dilated; their walls are regular, also at the level of the conical complete ureteral obstruction, which is surrounded by an area of irregular, dense, fibrotic tissue.

pulmonary lesions were discovered (Fig. 2A, B). The patient was submitted to an open procedure, including an ileal resection, a left hemicolectomy with colpo-hysterectomy, and salpingooophorectomy 'en bloc', followed by colorectal anastomosis, and temporary transverse colostomy. The postoperative period was smooth; histology confirmed an undifferentiated colonic adenocarcinoma infiltrating the uterine myometrium, staged as a T4NOMOG2. Adjuvant chemotherapy and closure of the colostomy were performed. After 1 year, a CT scan demonstrated thickening of the connective tissue in the lower retroperitoneum and in the pelvis, with only mild ureteral dilatation, but excluding signs of recurrent neoplastic disease. After 6 months, the patient presented an evident right uretero-hydronephrosis secondary to a complete obstruction of the pelvic ureter, which also appeared medially deviated (Fig. 2C, D). After a right percutaneous nephrostomy, a nephro-ureterectomy became necessary. Histology excluded any recurrent neoplastic disease, and only nonspecific ureteral and peri-ureteral fibrosis was demonstrated. The patient's subsequent follow-up to 2 years has been satisfactory.

\section{Discussion}

The mentioned cases have common characteristics: fibrotic obstruction of the ureter, following a nonspecific, long-standing retroperitoneal infection originating from a primitive intestinal pathology, already resolved, and a surgical procedure, performed with large dissection of the retroperitoneum. In particular, the primitive origin was an anastomotic fistula (case no. 1), and a spontaneous colovaginal fistula, secondary to a colonic cancer (case no. 2). The antineoplastic chemotherapy, present only in the case no. 2, can be recognized as an additional pathogenetic factor.

The above-mentioned pathological condition is absolutely different from the idiopathic retroperitoneal fibrosis, which extensively involves the retroperitoneum, usually beginning in its upper part as peri-aortic dense, greyish fibrous mass, and sometimes extended along the vena cava and the iliac arteries and veins, with medial deviation and extrinsic compression on the ureters. Clinical and radiological signs of chronic infection are absent; on the contrary, markers of auto-immunity are elevated; different autoimmune diseases and even inflammatory aneurysms of the abdominal aorta can be discovered. Pathohistology demonstrates a mixed fibrous and inflammatory tissue, developed especially in the perivascular spaces, rich in lymphocytes, plasma cells, macrophages, eosinophils, and fibrous component also containing myofibroblats (1).

These macroscopic and microscopic features were absent in our cases of chronic postinfectious retroperitoneal fibrosis, in which only nonspecific, fibrotic, and inflammatory lesions 


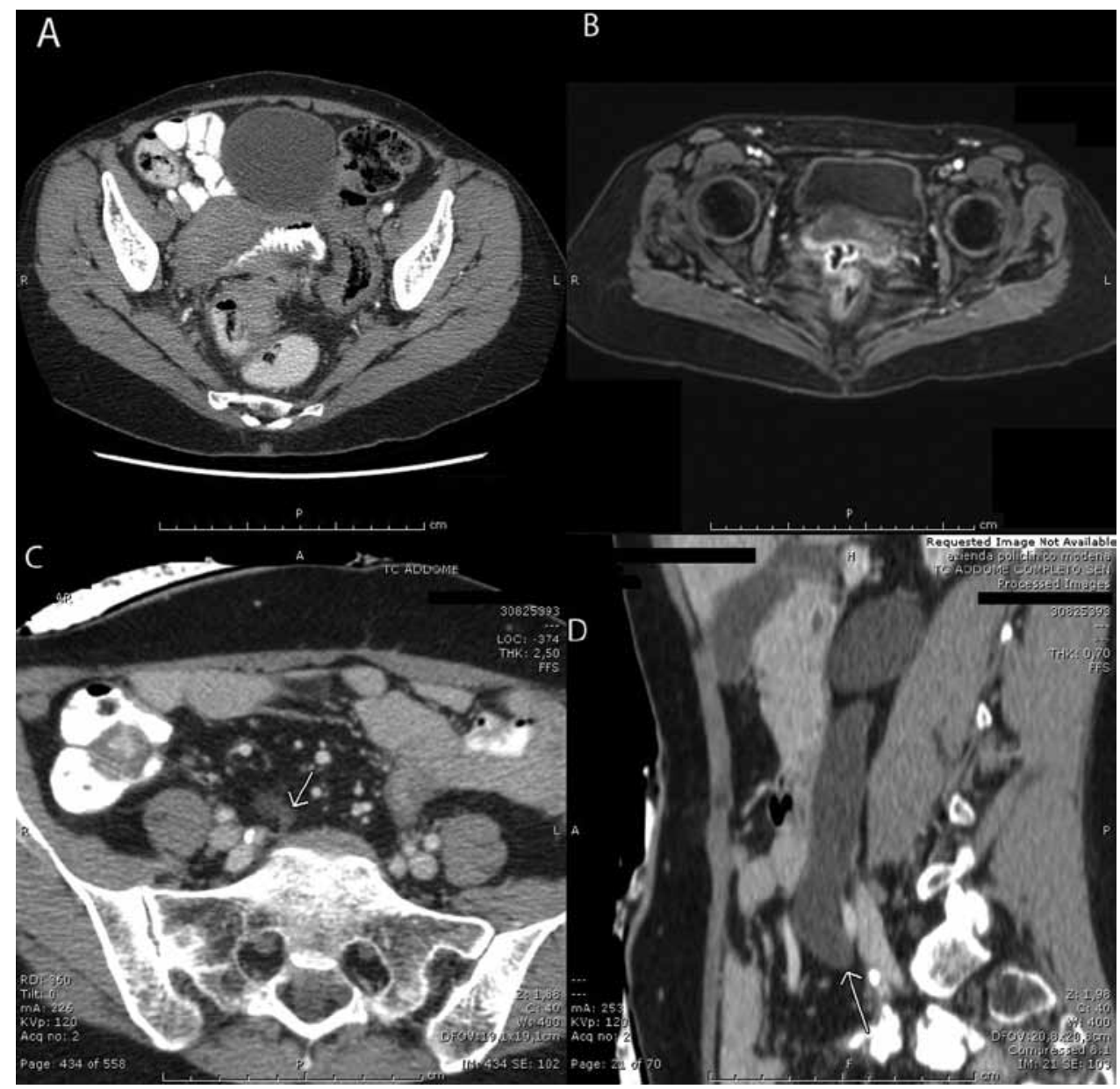

Fig. 2 - (A) Contrast-enhanced CT axial image. A neoplastic mass infiltrates the recto-sigmoid junction, surrounded by an inhomogeneous inflammatory tissue (arrow) and involving an ileal loop and the uterus. (B) Contrast-enhanced RM axial image. A fistulous tract runs toward the vagina (arrow). (C) Contrast-enhanced CT axial image. In the retroperitoneum, before the sacral promontory, an irregular area of dense fibrotic tissue extends toward the right iliac vessels (arrow); the ureter appears dilated. (D) Contrast-enhanced CT sagittal reconstruction. Right hydronephrosis with a dilated pelvis. The ureter, with completely regular walls, is enlarged in its upper and middle part, but with a lower complete obstruction (arrow).

of the ureter and of the surrounding connective tissue are present.

The modern imaging techniques, CT and MRI, are useful for detecting this particular pathology, enabling an accurate and complete study of the ureters and of the entire retroperitoneum, well appreciating the differences with the morphological characteristics of the idiopathic retroperitoneal fibrosis (10-13). In this last condition, the ureter, uni-laterally or bilaterally, without signs of other primitive pathology, is slightly medially deviated and especially narrowed or completely obstructed; the surrounding retroperitoneal connective tissue appears irregularly thickened and dense, while the vascular structures, venous and arterial, are not involved. Ultrasonography is a useful tool for an early detection and monitoring of the hydronephrosis, but it does not allow a complete study of the entire retroperitoneal or pelvic space.

The differential diagnosis of this disease involves, at first, other primary pathologies of the ureter, such as iatrogenic injuries, tuberculosis, and endometriosis, or of the retroperitoneum carcinomas, fibrosis secondary to radiotherapy, or common abscesses. Ureteral obstructions in course of Crohn's disease or of primitive arterial pathology are rare and usually incomplete (14-16).

Also, its clinical course is an important landmark, being the retroperitoneum involved primarily, and the ureter consequently.
From a pathogenetic point of view, we think that a postinfectious chronic inflammatory condition of the retroperitoneum acts on the ureteral and peri-uretereal tissues, and on their vascular and lymphatic network, inducing an inflammatory and then a fibrotic process. Timing of appearance, extension, and density of the fibrosis, evaluated by imaging techniques, can vary, being influenced by many factors, such as individual response, age, associated pathology, and therapy.

This disease is rare, and in the Medical Literature, we found reported only a similar case (17); on the contrary, a large series of complicated cases in colorectal surgery are referred.

This pathological condition is not even reported following an acute or chronic inflammatory pelvic gynecological disease; most probably, the infection only is not sufficient to give rise to it.

\section{Conclusion}

From a practical point of view, we underline the opportunity of a precocious and radical treatment of every retroperitoneal infection, especially if originated from a primitive intestinal pathology; a watchful attention to possible secondary ureteral lesions is necessary, remembering that the ureter is an organ particularly vulnerable and sensible to infection. 


\section{Disclosures}

Financial support: The authors have no financial disclosures to make. Conflict of interest: The authors have no conflict of interest.

\section{References}

1. Corradi D, Maestri R, Palmisano A, et al. Idiopathic retroperitoneal fibrosis: clinicopathologic features and differential diagnosis. Kidney Int. 2007;72(6):742-753.

2. Chambers WM, Mortensen NJ. Postoperative leakage and abscess formation after colorectal surgery. Best Pract Res Clin Gastroenterol. 2004;18(5):865-880.

3. Kingham TP, Pachter HL. Colonic anastomotic leak: risk factors, diagnosis, and treatment. J Am Coll Surg. 2009;208(2): 269-278.

4. Buchs NC, Gervaz P, Secic M, Bucher P, Mugnier-Konrad B, Morel $P$. Incidence, consequences, and risk factors for anastomotic dehiscence after colorectal surgery: a prospective monocentric study. Int J Colorectal Dis. 2008;23(3):265-270.

5. Kiran RP, da Luz Moreira A, Remzi FH, et al. Factors associated with septic complications after restorative proctocolectomy. Ann Surg. 2010;251(3):436-440.

6. Nisar PJ, Kiran RP, Shen B, Remzi FH, Fazio VW. Factors associated with ileoanal pouch failure in patients developing early or late pouch-related fistula. Dis Colon Rectum. 2011;54(4): 446-453.

7. Suzuki $H$, Ogawa $H$, Shibata $C$, et al. The long-term clinical course of pouchitis after total proctocolectomy and IPAA for ulcerative colitis. Dis Colon Rectum. 2012;55(3): 330-336.
8. Kiely JM, Fazio VW, Remzi FH, Shen B, Kiran RP. Pelvic sepsis after IPAA adversely affects function of the pouch and quality of life. Dis Colon Rectum. 2012;55(4):387-392.

9. Ahmed Ali U, Shen B, Remzi FH, Kiran RP. The management of anastomotic pouch sinus after IPAA. Dis Colon Rectum. 2012; 55(5):541-548.

10. Goenka AH, Shah SN, Remer EM. Imaging of the retroperitoneum. Radiol Clin North Am. 2012;50(2):333-355, vii.

11. Heller MT, Haarer KA, Thomas E, Thaete F. Acute conditions affecting the perinephric space: imaging anatomy, pathways of disease spread, and differential diagnosis. Emerg Radiol. 2012;19(3):245-254.

12. Lee SL, Ku YM, Rha SE. Comprehensive reviews of the interfascial plane of the retroperitoneum: normal anatomy and pathologic entities. Emerg Radiol. 2010;17(1):3-11.

13. Browne R F J, Zwirewich C, Torreggiani WC. Imaging of urinary tract infection in the adult. Eur Radiol. 2004;14(Suppl 3): E168-E183.

14. Manganiotis AN, Banner MP, Malkowicz SB. Urologic complications of Crohn's disease. Surg Clin North Am. 2001;81(1): 197-215, $x$.

15. Redman JF, Campbell GS. Ureteral obstruction secondary to iliac artery aneurysm. Urology. 1975;6(2):212-214.

16. Henriksen LO, Mejdahl S, Petersen F, Tøonnesen KH, Holstein PE. The incidence of ureteral obstruction secondary to aorto-femoral bypass surgery. A prospective study. Eur J Vasc Surg. 1988;2(6): 417-418.

17. Da Fonseca LM, De Souza Bechara C, Menezes CL, Corradi Fonseca CE, Silva RG. Rare complications after ileal pouchanal anastomosis: bilateral ureteral obstruction due to pelvic abscess and retroperitoneal fibrosis. Surgical Practice. 2012; 16(2):81-83. 